TRANS · núm. I8 2 20I 4

ARTICULOS · 199-214
La proliferación de nuevos diseños empíricos de investigación sobre los procesos cognitivos del traductor (Rodrigues, 2002), ha subrayado la necesidad de profundizar en el estudio de ciertas características individuales y de personalidad de los sujetos participantes, en un esfuerzo por dotar de sentido a la gran variabilidad registrada entre ellos (Jääskeläinen, 2000). Se revisan los antecedentes de la investigación sobre las diferencias individuales en el marco de nuestra disciplina y se presentan los conceptos básicos de la rama especializada de la psicología que se ocupa del análisis de las diferencias humanas. Por último, se esboza un marco metodológico preliminar para el estudio de las diferencias individuales en traductología, que podría propiciar el avance de esta área y el enriquecimiento de los diseños de investigación actuales.

PALABRAS CLAVE Diferencias individuales, traductología cognitiva, procesos cognitivos del traductor, metodología de investigación

\title{
La investigación de las diferencias individuales en traductología cognitiva
}

\author{
Alicia Bolaños Medina \\ Universidad de Las Palmas de Gran Canaria
}

\section{Individual Differences Research in Cognitive Translatology}

The increasing number of process-oriented empirical studies in translatology in recent years (Rodrigues, 2002), has highlighted the need to further analyze subjective and personality traits of research participants, in an effort to understand the high degree of variability detected among them (Jääskeläinen, 2000). Previous research on individual differences in translatology is reviewed prior to the introduction of the basic concepts underlying the specialized branch of psychology which studies human variability. Finally, a preliminar methodological framework which could foster the development of this area of study within our discipline and enrich current research study designs is presented.

Keywords Individual differences, cognitive traductology, process-oriented research, research methodology 


\section{INTRODUCCIÓN}

Aunque desde finales de los años sesenta la traductología ha dedicado gran parte de sus 200 esfuerzos a estudiar el espacio mental del traductor (Hurtado Albir, 20or), no fue hasta los años ochenta cuando se produjo la proliferación de nuevos diseños empíricos de investigación sobre el funcionamiento de sus procesos cognitivos (Rodrigues, 2002), tendencia que se ha consolidado en los últimos años (Fernández Sánchez y Muñoz-Martín, 20o8; O’Brien, 20II). Con ello, surgió también la preocupación académica sobre la metodología utilizada en un ámbito que prácticamente carecía de tradición empírica (Alves, 2003), y quedó patente la necesidad de mirar hacia otras disciplinas experimentales con mayor experiencia en este tipo de estudios; entre ellas, destacó desde muy temprano la psicología (Jääskeläinen, 20I2). La técnica de recogida de información tradicionalmente más utilizada han sido los protocolos de verbalización del pensamiento en voz alta o TAP (Think-Aloud Protocols), que, en sus muchas variantes, recogen las introspecciones de los participantes en las investigaciones sobre una tarea de traducción. Los resultados de dichos trabajos pronto pusieron de manifiesto la necesidad de profundizar en el estudio de ciertas características individuales y de personalidad de los sujetos participantes, en un esfuerzo por dotar de sentido a la gran variabilidad registrada entre ellos (Jääskeläinen, 2000).

Por un lado, a pesar de haberse hallado ciertos patrones comunes entre los traductores profesionales, frente, por ejemplo, a los estudiantes de traducción, también se concluyó que los procesos mentales de cada traductor son únicos, difícilmente sistematizables en categorías estancas (Asadi y Séguinot, 2005). Por el otro, quedó patente que había que controlar en mayor medida las variables extrañas de tales diseños de investigación para aumentar su rigor metodológico; era necesario establecer mejor el perfil de los sujetos y analizar hasta qué punto ciertos factores individuales podían ser responsables de los resultados hallados (Hansen, 2003; MuñozMartín, 2009). En este contexto, incorporar la experiencia metodológica de la psicología de las diferencias individuales en los modelos traductológicos de investigación no solo cobra pleno sentido, sino que también puede servir para organizar los esfuerzos académicos en torno a un marco metodológico común capaz de promover el avance del conocimiento en esta área de forma más sistemática.

El objetivo de este artículo no es solo mostrar el interés de este campo, sino también esbozar un marco metodológico preliminar para el estudio de las diferencias individuales en traductología y propiciar así el avance y el enriquecimiento de los diseños de investigación actuales. Para ello, se revisarán los antecedentes empíricos y no empíricos de la investigación sobre las diferencias individuales realizada en el marco de nuestra disciplina. A continuación, se presentarán los conceptos básicos de la rama especializada de la psicología que tradicionalmente se ha ocupado del análisis de las diferencias humanas, para abordar más tarde, desde un enfoque traductológico, los objetivos, las fases, los tipos de variabilidad, las unidades de análisis y los diseños de investigación propios de este ámbito. Por último, se recogerán las principales conclusiones obtenidas.

\section{ANTECEDENTES DEL ESTUDIO DE LAS DIFERENCIAS INDIVIDUALES EN TRADUCTOLOGÍA}

Los antecedentes de este campo se pueden dividir según dos criterios: si analizan la personalidad del traductor en su conjunto o solamente 
algunas diferencias individuales más concretas; y si se trata de enfoques empíricos o no.

La primera mención formal a la influencia de la personalidad del traductor sobre el producto de su trabajo se la debemos a Reiss ([1971] 2000), quien la equipara en importancia a la del autor del texto original. Para ello, se fundamenta en la disciplina de la caracterología, que abarca los estudios sobre las peculiaridades que conforman el carácter y la personalidad de los seres humanos, en especial sobre la especificidad de las diferentes variedades de individuos frente a lo que se puede considerar originalmente personal en ellos. Reiss adopta la clasificación de seis tipos básicos de personalidad de Spranger (I920), quien define el carácter como el conjunto de actos o vivencias referidos a la cultura. Esta se concreta en función de la ciencia, el arte, la economía, la religión, etc. y en cada una de estas ramas se tiende hacia unos valores dados, que determinan los tipos de individuos propuestos por este autor: teórico, económico, estético, social, agresivo y religioso. Reiss va más allá al postular la idoneidad de cada traductor para la traducción de un tipo de textos, según reúna las características propias de una u otra categoría. Por ejemplo, el tipo estético tendrá facilidad para trabajar con textos expresivos, el tipo teórico preferirá los textos técnicos y el tipo económico sería el más adecuado para la traducción de textos apelativos.

Otro enfoque no empírico digno de mención es el adoptado por Barboni (1999). Desde la perspectiva del psicoanálisis, analiza los mecanismos potenciales de defensa del traductor ante la situación de estrés que supone todo acto traslativo. Entre otros, distingue la identificación del traductor con el autor del texto original hasta el punto de no discernir los límites entre la creación de este último y la suya propia (en este sentido, Barboni habla de «traductor caníbal»).
También estudia la inhibición que se produce cuando el traductor intenta dejar fuera de su conciencia todas las representaciones, suscitadas por el texto, que juzga inaceptables según sus valores y pulsiones (entendidas, en sentido freudiano, como formas de energía psíquica profunda que orientan el comportamiento hacia un fin, a menudo relacionadas con la muerte, el sexo o la violencia). Pero quizá el más destructivo para la traducción sea el mecanismo de sublimación, ya que el traductor se siente retraído o inhibido por un respeto demasiado grande hacia el autor y se ve incapaz de tomar la distancia necesaria para realizar su trabajo con éxito. Según Barboni (1999), en el pasado los traductores se resistían a profundizar en sus procesos psicológicos y daban la impresión de no querer estropear la ilusión de magia que suele revestir su trabajo. La conducta del traductor ante una dificultad está muy ligada a su personalidad y, de nuevo, encontramos la idea de que de ella depende que se trate de un profesional más o menos adecuado para la traducción de ciertos tipos de textos.

Tanto el enfoque de Reiss como el de Barboni tienen el mérito de haber hecho hincapié en la influencia de la personalidad del traductor en su conjunto sobre el resultado del proceso, pero ambos adolecen de un carácter marcadamente teórico, alejado del rigor de la metodología empírica necesaria para su verificación.

Tuvieron que pasar dieciséis años desde la primera alusión a la importancia de la personalidad del traductor para que se realizara el primer estudio sistemático en esta línea. Con el objetivo de comparar la personalidad de los traductores y los intérpretes profesionales, Henderson (I987) utilizó el cuestionario factorial de personalidad de I05 ítems I6PF (Cattell, 1946) en una muestra de 100 profesionales (65 traductores y 35 intérpretes) y 45 estudiantes. Los resultados de su trabajo establecen la existencia 
de ciertos patrones diferenciales, pero son muy atenuados. Las discrepancias entre unos y otros son mucho menos marcadas de lo que cabría esperar; así, la ansiedad es un rasgo compartido por ambos perfiles profesionales, aunque se había hipotetizado lo contrario, pero también un factor diferencial frente al grupo de estudiantes, que obtuvo en su conjunto una puntuación menor que la de los traductores e intérpretes profesionales. Entre las diferencias más marcadas se encuentran las siguientes: por un lado, los traductores son más reservados y más inteligentes que los intérpretes, mientras que estos últimos son más estables emocionalmente que los primeros. Por el otro, los traductores tienden a ser más prácticos y los intérpretes más imaginativos.

De nuevo, encontramos que hasta veinte años más tarde no se volvió a utilizar un test de personalidad en el marco de una investigación sobre traducción. Fue Hubscher-Davidson (2007) quien, para determinar su influencia en el proceso y el resultado, aplicó a 20 sujetos el test de personalidad Myers-Briggs, de 72 ítems, elaborado a partir de las teorías de Jung (I92I). Las dimensiones aplicadas son «introvertido» o "extravertido», según el interés del individuo esté volcado prioritariamente en el mundo interior o en el exterior; «sensorial» o «intuitivo», en función de si se orienta hacia la percepción de hechos reales y prácticos o a la toma de conciencia de relaciones y posibilidades; «racional» o "emocional», dependiendo de si dominan la razón y el pensamiento o el sentimiento y las opiniones personales y subjetivas a la hora de tomar decisiones; y, por último, "calificador" o "perceptivo", si se vive de forma planificada y resistente al cambio o de forma espontánea. De la combinación de estas dicotomías se obtienen i6 tipos psicológicos diferentes.
Hubscher-Davidson (2007; 2009) halló que los sujetos más intuitivos, y aquellos que pertenecían a tipos de personalidad con un componente de intuición importante, obtuvieron mejores resultados que los sensoriales, lo que parece indicar que una aproximación relativamente abstracta resulta más adecuada. La combinación de individuos de carácter sensorial con una inclinación racional o calificadora ocasionó un bajo rendimiento a la hora de traducir, aunque, entre diferentes individuos pertenecientes a un mismo tipo, un nivel de confianza elevado parece ser determinante para el rendimiento final. En cualquier caso, más que la asociación con un determinado criterio, la clave para obtener un producto final de calidad parece residir en el equilibrio que se produce entre varios de ellos.

La relevancia de la aportación de HubscherDavidson viene dada, en primer lugar, por haber demostrado empíricamente que la personalidad del traductor influye en su actitud, su rendimiento y en la calidad del TM. En segundo lugar, los resultados obtenidos pueden ser aplicados para optimizar la formación de traductores. Por último, también ha ayudado a consolidar esta reciente vía de investigación. Sin embargo, la validez de la prueba psicométrica utilizada en el experimento ha sido puesta en duda (McCrae y Costa, I989) y se ha llegado incluso a proponer como un claro ejemplo del efecto Forer (I949); es decir, de cómo los sujetos aceptan de buen grado ciertas descripciones de personalidad que se supone que han sido elaboradas específicamente para ellos, pero que en realidad son lo suficientemente vagas como para poder ser aplicables casi a la mayoría de las personas. Por ello, sería interesante replicar el estudio y aplicar esta vez un instrumento con propiedades psicométricas más adecuadas a un mayor número de sujetos. 
Otra investigación diseñada para analizar la relación entre la personalidad del traductor y la calidad final de su traducción de textos narrativos ha sido llevada a cabo por Amirzehni (2013). Para ello aplicó a 30 estudiantes de traducción el inventario de personalidad NEO, que mide cinco rasgos: neuroticismo, extroversión, apertura a la experiencia, amabilidad y responsabilidad. La principal conclusión de la investigadora fue que no parecía existir una relación clara entre ambas variables. ${ }^{\mathrm{I}}$

Muchos son los autores que han subrayado la vinculación de ciertas características personales con la competencia traductora y la calidad del resultado final. Este interés ha provocado la aparición en los últimos años de otras investigaciones que, si bien no analizan de forma estructural la personalidad del traductor, sí profundizan en la descripción de algunas dimensiones relevantes para la traductología cognitiva y que son objeto potencial de análisis de la psicología de las diferencias individuales. Entre estas variables destacan: la tolerancia al estrés (Jääskeläinen, 200o); la flexibilidad, el realismo y la curiosidad intelectual (Kussmaul y Tirkonnen-Condit, I995); la autoconfianza (Laukkanen, I996; Froeliger, 2004; HjortPedersen y Faber, 2009); el miedo a errar y la pasividad (Laukkanen, I996; Jääskeläinen, 2000); la creatividad (Kussmaul, I995); el autoconcepto (Kiraly, I997); la caracterización de sujetos en cuanto a sus capacidades cognitivas,

\footnotetext{
I Los datos han sido extraídos del resumen de la comunicación titulada «The Relationship between the Translators's Personality and the Quality of Translation in Narrative Texts», que ha sido presentada en la International Conference on Interdisciplinary Translation Studies, celebrada del 30 abril al 2 de mayo de 2013 (es decir, en las mismas fechas en las que se finaliza el presente artículo) en la universidad Imam Reza Int'1 (Irán). A pesar de haber intentado ponernos en contacto con la autora, no ha sido posible acceder a la totalidad del texto para valorar mejor su aportación.
}

en particular la de memoria, la de procesamiento de la información y la de comprensión verbal (Bajo et al., 20or; Muñoz-Martín, 2009); la competencia traductora (Beeby et al., 20II); la 203 tolerancia a la ambigüedad (Tirkkonen-Condit, 200o; Angelone, 20I0; Angelone y Shreve, 20Ir; Bolaños-Medina, 2013); la afectividad negativa, la estabilidad emocional y las estrategias de afrontamiento (Bontempo y Napier, 2orI; Bontempo y Loggerenberg, 20II); la inteligencia emocional (Hubscher-Davidson, 2013); la eficacia colectiva y la autoeficacia (BolañosMedina, 2008; 2013); la tendencia o aversión al riesgo (House, 2000; Künzli, 2004; Pym, 2005); e incluso la propensión al uso de ciertas estrategias de toma de decisiones (Tirkkonen-Condit, 1996; Wilss, 1996; Prassl, 2010) y de traducción (Asadi y Séguinot, 2005).

Aunque no cabe duda del interés de estos estudios, son varios los factores que, en general, dificultan la sistematización del conocimiento que de ellos se puede derivar. Aunque no afecten los que enumeramos a continuación en igual medida a todos, dadas las limitaciones de longitud del presente trabajo, los presentaremos de forma global para facilitar su comprensión.

- Se distingue cierta atomización en las investigaciones; en ocasiones se presentan resultados de forma casi anecdótica, aislada, si bien muchas de las variables analizadas están relacionadas y se podrían estudiar conjuntamente.

-El número de sujetos a menudo resulta insuficiente, son pocas las investigaciones en las que participa una muestra representativa de la población, y (quizá debido al uso de los protocolos de verbalización de pensamiento, cuya metodología de análisis, de carácter cualitativo, resulta bastante laboriosa) en muchos casos se incluyen apenas entre uno y diez sujetos (Rodrigues, 2002). 
-Poco uso de instrumentos psicométricos, quizá por desconocimiento de su existencia. A la hora de la recogida de datos, las pruebas psicométricas son procedimientos que facilitan la caracterización de los sujetos y la descripción de determinadas conductas. ${ }^{2}$

- Se trata de estudios difícilmente comparables. La atomización de estas investigaciones hace que a menudo se lleven a cabo en condiciones muy diferentes, lo que dificulta su comparación, tanto en la fase de contraste de resultados por parte de otros investigadores, como a la hora de realizar metaanálisis cualitativos y cuantitativos más rigurosos.

-E1 control experimental resulta con frecuencia escaso. El investigador debe velar por que las únicas fuentes de variación de su experimento sean las que ha establecido en su diseño experimental, sin que actúen variables extrañas que resten validez a sus resultados. Por ejemplo, las diferencias individuales pueden actuar como variables extrañas, no relacionadas directamente con el propósito del estudio, y que habrá que controlar.

-En su mayoría son estudios descriptivos. La fase de descripción es quizá la más importante, pero a medida que los conocimientos se van consolidando en una disciplina, lo lógico es que se intente avanzar hacia la explicación y la predicción. La traducción no tiene por qué ser una excepción.

-Poco uso del potencial estadístico en general y, sobre todo, del ofrecido por los últimos avances en esta disciplina. Del aprovechamiento adecuado de las herramientas estadísticas dependerá también en gran medida la transición hacia fases explicativas y predictivas.

2 Permiten el control de las variables extrañas dependientes del sujeto y se pueden clasificar en autoinformes, observación, técnicas subjetivas, técnicas proyectivas y técnicas objetivas (Bolaños-Medina, 20I2).
-Escasa triangulación de datos. Si bien es cierto que en los últimos años se ha constatado cierta preocupación por la diversificación de los métodos de recogida de datos de un mismo estudio (Alves, 2003), todavía se deben realizar mayores esfuerzos en este sentido que reviertan en una mayor fiabilidad de los resultados.

-No se suelen replicar las investigaciones para contrastar los resultados.

- Se trasluce una falta de marco común de investigación capaz de articular y dotar de coherencia a los esfuerzos realizados.

\section{FUNDAMENTOS DE LA PSICOLOGÍA DE LAS DIFERENCIAS INDIVIDUALES}

Desde hace siglos, los seres humanos se han interesado por la diversidad de su comportamiento. La diferencias individuales afectan a todas las áreas del complejo funcionamiento psicológico de nuestra especie (Royce y Powell, I983), de ahí el interés de su estudio para disciplinas tan dispares como la cardiología (BoothKewley y Friedman, i987) o el aprendizaje de lenguas extranjeras (Skehan, I99I). Teorías de la época clásica tales como la propuesta por Hipócrates sobre el temperamento humano, la de Galeno sobre la relación entre los humores y las diferencias individuales o la de Teofrasto acerca de los caracteres, son los antecedentes más antiguos de los que tenemos referencia (Olmedo Montes y Sánchez-Elvira Paniagua, 2003). Este interés fue retomado en nuestro país durante el Renacimiento por Juan Huarte de San Juan ([I575] I976), hoy reconocido como precursor de esta rama de la psicología, quien estableció una descripción exhaustiva de caracteres así como una serie de métodos dirigidos a entrenar capacidades básicas. Sin embargo, el acontecimiento teórico y metodológico que impulsó definitivamente el desarrollo de la disciplina en 
el marco de las ciencias fue la confluencia de la teoría darwinista de la evolución (Darwin, [1872] i998) y la aportación del británico Galton (1883). Fue este último quien desarrolló el modelo y el objeto de estudio de las diferencias individuales, elaboró métodos y pruebas para su evaluación objetiva y llevó a cabo los primeros análisis estadísticos. Tres escuelas principales impulsaron su andadura como ciencia: la inglesa, fiel al legado de Galton y con Spearman (I9I4) como principal exponente y precursor del análisis factorial actual; la estadounidense de Cattel (I946), quien introdujo los test mentales de naturaleza normativa con vistas a un mejor aprovechamiento de los recursos humanos y docentes; y la francesa, centrada en el estudio de los procesos mentales superiores a partir de la conducta (Binet y Simon, I954).

La psicología de las diferencias individuales se encarga de la «descripción, predicción y explicación de la variabilidad interindividual, intergrupal e intraindividual con respecto a su origen, manifestación y funcionamiento» (Sánchez-Elvira Paniagua y Olmedo Montes, 2003: IO7). La psicología de la personalidad se interesa por la configuración de las funciones y los procesos integrados en una imagen unitaria, en lo que hace a cada individuo; la psicología de las diferencias individuales estudia, desde un enfoque elemental y de forma aislada, «los atributos que luego son agregados a una estructura global que corresponde, de hecho, a la totalidad del individuo» (op.cit., 2003: I39).

$\mathrm{E} 1$ enfoque de las diferencias individuales, como cualquier otro de carácter científico, se plantea una serie de preguntas, entre ellas qué propiedades psicológicas es necesario analizar para describir a un individuo, cómo actúan estas, cómo se relacionan con el funcionamiento biológico y cómo se originan genética y ambientalmente (Colom Marañón, I998).
Una preocupación constante a lo largo del desarrollo de esta disciplina, y que contribuye a delimitar sus etapas evolutivas, ha sido en qué medida se puede afirmar que existe un comportamiento relativamente estable en el sujeto, tanto intraindividual como interindividualmente (Sánchez Cánovas y Sánchez López, r999). Entre finales del siglo XIX y principios del XX, el interés giró en torno a la estabilidad temporal y situacional de la conducta y surgió el concepto estático de «rasgo», quizá históricamente el más importante de esta disciplina, al que se atribuyen ciertas características que resumimos a partir de Colom Marañón (I998: I22-I25):

-Son disposiciones, más o menos determinadas por la herencia y el medio ambiente, que facilitan la descripción de las personas, la predicción de su conducta y la explicación de por qué es consistente (Eysenck y Eysenck, 1985).

-Permiten situar a los individuos en una serie de dimensiones continuas y se establecen predominantemente mediante metodología correlacional.

- Las respuestas a las preguntas de las pruebas psicométricas son signos o síntomas de los rasgos de los individuos y ayudan a contrastar una determinada teoría psicológica.

-La investigación psicológica ha estudiado tres categorías: los intelectuales (capacidad de razonar, de memoria o de lectura), los temperamentales (por ej., extroversión o estabilidad emocional) y los motivacionales (actitudes, valores o intereses).

En un segundo periodo, la mayor preocupación de los científicos fue la consistencia y se enfatizaron las variables de la situación frente a las de la persona: si la conducta es más específica respecto a cada situación que constante entre diferentes situaciones, es posible establecer nexos causales entre los patrones de respuesta y los estímulos de la situación; el método más 
adecuado para descubrirlos es el experimental (Bowers, I973). Por último, la tercera fase recoge enfoques que implican distintas concepciones de la relación persona-situación y difieren en su aproximación metodológica y de análisis de los datos. En efecto, tanto el enfoque centrado en la persona como el basado en la situación se ven desplazados por el interaccionismo, que estudia la acción recíproca, dinámica y constante entre la persona y la situación (Sánchez Cánovas y Sánchez López, I999). Comparte ciertas características con la perspectiva de los rasgos, en tanto en cuanto admite la existencia de variables latentes que influyen en la conducta, pero las considera como «facetas independientes que forman parte de un sistema interno mediante el cual el sujeto empareja la información proporcionada por la situación con la forma característica de responder que tiene el individuo» (Sánchez Cánovas y Sánchez López, ı999: 85). Para Magnusson y Endler (I977), el interaccionismo establece que el sujeto es un agente activo e intencional cuyos factores cognitivos y motivacionales determinan esencialmente la conducta; esta última es una función de un proceso de retroalimentación continua entre el individuo y la situación, cuyo significado psicológico para el sujeto es determinante.

\section{HACIA UN MARCO METODOLÓGICO PRELIMINAR PARA LA INVESTIGACIÓN DE LAS DIFERENCIAS INDIVIDUALES EN TRADUCTOLOGÍA}

Para lograr su pleno desarrollo científico, la investigación sobre diferencias individuales en traductología debe sustentarse en un marco metodológico capaz de orientar los esfuerzos académicos hacia una meta común. A continuación esbozamos ciertos fundamentos, inspirados en la experiencia acumulada durante muchos años por la psicología, que podrían servir de punto de partida para el avance de este campo.

\section{I. Objetivos, fases y tareas}

En consonancia con los tres objetivos principales que persigue la psicología de las diferencias individuales recogidos en su definición, se pueden distinguir otras tantas fases bien delimitadas, cada una de las cuales se corresponde con ciertas tareas, tal y como se muestra a continuación siguiendo a Sánchez-Elvira Paniagua y Olmedo Montes (2003).

Durante la fase de descripción, predominan dos cometidos. En primer lugar, la observación de las conductas en muestras representativas de la población y su evaluación mediante instrumentos de recogida de datos de propiedades psicométricas adecuadas. La elección de herramientas adecuadas resulta crucial. Si bien en la actualidad existe en este campo una gran variedad de herramientas informatizadas (como las de captura de pantalla y de seguimiento del globo ocular), y de instrumentos psicométricos de validez y fiabilidad contrastadas (Kline, I996; Bolaños-Medina, 2012), también es posible desarrollar otros nuevos adaptados al objeto de estudio específico de nuestro campo, algo que en sí constituiría una interesante vía de investigación apenas explorada. ${ }^{3}$

En segundo lugar, la clasificación de las dimensiones halladas en un número restringido de categorías predictivas que representen un volumen mayor de comportamientos diferentes, mediante métodos correlacionales y de análisis

3 Con la excepción de ciertos cuestionarios, como por ejemplo los retrospectivos en los protocolos de verbalización del pensamiento, los de carácter sociolingüístico, como el del grupo PETRA (Marín García 2008: 63) o los cuestionarios de problemas y sobre conocimientos de traducción del grupo de investigación PACTE (Presas Corbella, 2006). 
factorial. La unidad de estudio propia de esta fase es el rasgo. Lo importante en este punto es que «esas entidades o constructos hipotéticos y no directamente observables se formulen y relacionen con fenómenos de carácter empírico, más o menos observables, de forma lo suficientemente precisa como para posibilitar la deducción de enunciados contrastables, así como la elaboración posterior de teorías científicas» (Sánchez-Elvira Paniagua y Olmedo Montes, 2003: 108). Una revisión bibliográfica concienzuda sobre el área concreta de estudio nos llevará a concluir que buena parte del trabajo de esta fase ya ha sido realizado anteriormente desde la psicología, cuya aportación será un valioso punto de partida.

Se trata también de identificar las dimensiones que en mayor medida se relacionan con la conducta del traductor y que, por lo tanto, deberán tenerse en cuenta, ya sea como variables independientes o como variables extrañas que debemos controlar para incrementar la validez de nuestros resultados. Este tipo de esfuerzos podrían contribuir a paliar factores tales como la atomización, el escaso control experimental o el bajo grado de comparatividad que caracterizan a muchos estudios actuales.

En la fase de predicción se analiza la relación de las dimensiones de estudio con el rendimiento y se establece su carácter predictivo, útil por ejemplo en los ámbitos académico o laboral. Es propio de esta fase el uso de modelos estadísticos de regresión.

Por último, en la fase de explicación, se elaboran, en primer lugar, teorías explicativas a partir de la naturaleza y funcionamiento de las dimensiones de análisis y los procesos implicados, para luego evaluarlas. Para esta última tarea, como veremos más adelante, se pueden utilizar correlaciones causales y ecuaciones estructurales.

\subsection{Tipos de variabilidad estudiada}

Se pueden estudiar tres tipos diferentes de variabilidad. La interindividual se encarga de analizar la diversidad de comportamientos de sujetos distintos de una población en un momento y circunstancias dados; se evalúa «la posición que ocupa el sujeto en relación con los demás al compararlos en los diferentes rasgos, [...] la situación comparativa del individuo respecto a su grupo» (Sánchez Cánovas y Sánchez López, 1999: 72). En este marco se llevan a cabo, por lo tanto, estudios transversales de ciertos constructos con cierta consistencia transituacional (que se manifiestan en distintos tipos de situaciones), estabilidad temporal (patrones que aparecen en varios momentos temporales o se mantienen invariables durante un lapso relativamente duradero) y relevancia psicológica. Un ejemplo de investigación que analiza este tipo de variabilidad sería el estudio del autoconcepto en una muestra de estudiantes universitarios de traducción, por ejemplo para evaluar posteriormente su relación con su rendimiento al traducir.

La variabilidad intraindividual permite dos aproximaciones: una transversal, que examina el perfil psicológico o la estructura de personalidad de los sujetos, la «configuración propia que, en interacción con la situación ambiental, da lugar a las diversas expresiones conductuales del individuo, de carácter normalmente reversible, o diferencias intraindividuales de carácter sincrónico, que pueden cambiar o modificarse» (Sánchez-Elvira Paniagua y Olmedo-Montes, 2003: IIo); y otra longitudinal o a lo largo del tiempo, si bien es esta última la más interesante en tanto en cuanto permite analizar la evolución de la estructura, organización y dinámica particulares de sus características psicológicas y eliminar el llamado efecto de cohorte o generacional. Resulta útil, por ejemplo, en contextos didácti- 
cos, y el lapso temporal puede ir desde periodos de larga duración, identificados por ejemplo con las diferentes etapas de la vida, hasta cambios 208 pasajeros, siendo a menudo los periodos intermedios los más interesantes (Sánchez Cánovas y Sánchez López, I999). Consideremos, por ejemplo, el estudio de la evolución de la autoeficacia de un estudiante de traducción desde su inicio de la formación hasta su conclusión, o posteriormente desde sus primeros pasos a su consolidación como profesional en el mercado de la traducción.

El tercer tipo de variabilidad analiza las diferencias entre grupos, ya sean estos naturales, formados sin intervención del experimentador (por ejemplo, según variables tales como la edad, el sexo, etc.), o configurados por el investigador (ad hoc). En este último caso, cualquier atributo psicológico mesurable desde la perspectiva interindividual podría actuar como variable de agrupación. En este nivel de análisis, la comparación de grupos extremos (por ejemplo, el $15 \%$ de puntuación superior con el mismo porcentaje inferior) en un rasgo determinado (por ej., la ansiedad), o el contraste de las características distintivas de profesionales y novatos en la organización del proceso traductor o en una dimensión dada, se encuentran entre los diseños que podrían arrojar datos de interés.

\subsection{Variables de análisis}

Las unidades de análisis pueden ser disposicionales o procesuales (Sánchez-Elvira Paniagua y Olmedo Montes, 2003). Las unidades disposicionales, representadas por el concepto de rasgo, han sido tradicionalmente las más estudiadas. Ejemplos de rasgos son la asertividad, la apertura a la experiencia o la introversión (Henderson, 1987). Como hemos visto, se caracterizan por ser disposiciones latentes inferidas, con estabilidad temporal, consistencia transituacional, amplitud y carácter dimensional; es decir, se operativizan como dimensiones cuantitativas, así que a mayor puntuación en un rasgo, «mayor probabilidad de ocurrencia de las conductas hacia las que predispone» (Sánchez-Elvira Paniagua y Olmedo Montes, 2003: I3I), mayor frecuencia y mayor intensidad de la respuesta. Tradicionalmente, en el ámbito de la inteligencia, se utiliza otra terminología para denominar a los rasgos. Es el caso de las aptitudes, o capacidades potenciales para realizar actos no aprendidos, y de las habilidades, o destrezas adquiridas mediante entrenamiento y experiencia. Así, se puede analizar la capacidad verbal de los individuos, pero también su habilidad lectora, ambas de interés para la traducción.

Las unidades procesuales de análisis se requieren en la fase explicativa de la investigación en diferencias individuales, ya que es necesario abordar los mecanismos funcionales que provocan la variabilidad comportamental de los individuos. Siguiendo a Mischel y Shoda (I995), si atendemos a la relación del individuo con el mundo externo, la unidad fundamental es la interacción de la persona con la situación. En cambio, si nos detenemos a valorar el nivel interno, estudiaremos los procesos cognitivos, emocionales, motivacionales y las estrategias de afrontamiento de situaciones concretas.

El estudio de la interacción persona-situación implica el análisis de los tipos de situaciones que de forma consistente elicitan cada disposición; los patrones significativos observables en la interacción de la persona con el ambiente; y la evaluación de las probabilidades y la frecuencia de cambio del comportamiento en respuesta a ciertas configuraciones situacionales (Sánchez-Elvira Paniagua y Olmedo Montes, 2003). Sin embargo, cada vez se tiene más en cuenta el significado subjetivo de la situación y 
sus características realmente relevantes:

However, if situation units are defined in terms of features salient for the researcher, but trivial for, or irrelevant to, the individuals studied, one cannot expect their behaviors to vary meaningfully across them, and the resulting pattern of behavior variation therefore would be unstable and meaningless. To discover the potentially predictable patterns of behavior variability that characterize individuals, the first step is to identify those features of situations that are meaningful to them and that engage their important psychological qualities (e.g., personal constructs and goals). (Mischel y Shoda, I995: 250)

Supongamos por ejemplo el estudio de la conducta de estudiantes en una traducción contrarreloj, frente a una prueba de traducción sin límite estricto de duración, ambas con grabación en vídeo de los sujetos. En la primera situación, la conducta de estrés del traductor podría deberse tanto a la premura con la que debe trabajar como al hecho de que queden registrados todos sus gestos y acciones. Dependiendo del sujeto, es probable que uno de estos dos factores tenga mayor peso en su conducta que el otro, de ahí el interés en conocer exactamente qué características de la situación son relevantes para cada individuo.

Además de la unidad de análisis personasituación ya tratada, en el nivel interno cabría estudiar (Sánchez-Elvira Paniagua y Olmedo Montes, 2003):

-Procesos cognitivos o unidades elementales de funcionamiento mental, tales como la percepción, la memoria, la forma en que se adquiere información, los estilos cognitivos, o los estilos de traducción (Carl et al., 20II), entre otros.

-Procesos emocionales y motivacionales. En el ámbito de la traducción, se inscribirían aquí por ejemplo los estudios sobre afectividad negativa y estabilidad emocional de Bontempo y Napier (20II) o sobre inteligencia emocional (Hubscher-Davidson, 20I3).

-Estrategias o programas de acción que dominar un tipo de tarea. En la fase explicativa, se trata de analizar de qué forma dichas estrategias originan diferencias patentes en el comportamiento de los individuos. Es el caso de los procesos controlados de resolución de problemas o toma de decisiones.

Por su parte, desde una perspectiva más amplia, Mischel y Shoda (I995) definen cinco unidades cognitivo-afectivas de la personalidad, que fácilmente podrían plasmarse en otras tantas variables de análisis útiles en traductología que reflejamos a continuación a partir del esquema de Sánchez-Elvira Paniagua y Olmedo Montes (2003: 138): codificaciones externas e internas (sobre nosotros mismos, otras personas y situaciones); expectativas y creencias; afectos o emociones; metas y valores (acontecimientos y estados afectivos deseables y no deseables) y competencias y planes de autoregulación o guiones desarrollados por el individuo para organizar la acción.

\subsection{Diseños de investigación}

No es el objetivo de este apartado profundizar en el funcionamiento de todos los diseños de investigación posibles (Fontes de Gracia et. al., 20Io), ni siquiera de los más utilizados en traducción (Rojo López, 2013), sino más bien destacar ciertas características de los más útiles para el estudio de las diferencias individuales del traductor.

Los diseños «ex-post-facto» son los más utilizados habitualmente en esta área para analizar las interdependencias entre las variables y permiten realizar inferencias a partir de la variación conjunta. El investigador no tiene control sobre 
variables, ni manipula ni modifica las variables independientes, ya sea porque no son manipulables o porque ya acontecieron. Tampoco es 210 posible la asignación aleatoria de los sujetos, que son seleccionados según posean o no ciertas características. No permiten el análisis causal pero tradicionalmente en ellos se distinguen también una variable dependiente (constructo que debe explicarse) y una independiente (la que tiene valor explicativo). Este tipo de diseños se divide a su vez en correlacionales y propiamente comparativos.

Si nuestro objetivo es conocer el entramado de relaciones que subyace al fenómeno estudiado, es necesario optar por un diseño correlacional; si perseguimos identificar los aspectos que diferencian unos grupos de estudio de otros, cabría usar un diseño comparativo (Sánchez Cánovas y Sánchez López, I999). Los diseños correlacionales ayudan a explorar y estructurar un campo de observación. Cuando su objetivo es descriptivo, permiten conocer relaciones entre variables de interés y analizar estructuras subyacentes. En este último caso se utilizan análisis multivariados o análisis factorial, análisis de cluster y escalas multidimensionales.

El análisis factorial ha sido una de las técnicas más usadas para estudiar las diferencias individuales. Se fundamenta en que «los innumerables factores que intervienen en la producción de una actividad psicológica no actúan separados, ni se organizan al azar, sino que ante los mismos problemas tienden a actuar organizados [...] y dirigidos de una cierta manera que les presta unidad funcional» (Andrés Pueyo, I997: I34). Se trata de simplificar, es decir: a partir de un número elevado de variables, se pretende hallar factores que impliquen cierta estructuración interna y que sean capaces de explicar una variedad de resultados en diferentes pruebas. Por ejemplo, la investigación en inteligencia ha hallado que la gente que obtiene una nota alta en una prueba de habilidad verbal también rinde bien en otras que requieren habilidades verbales. Los investigadores explican esto mediante el uso del análisis factorial para aislar un factor (inteligencia cristalizada o verbal), que representa el grado de capacidad para resolver problemas usando habilidades verbales (Amor Andrés y SánchezElvira Paniagua, 2003).

Cuando su finalidad es predictiva, se usan modelos de regresión (simple, múltiple y de análisis discriminante). Se trata de técnicas estadísticas para explorar y cuantificar la relación entre una variable dependiente o criterio y una o más variables llamadas independientes o predictoras y desarrollar una ecuación lineal con fines predictivos. Los valores de las variables predictoras se fijan de antemano (Sánchez Cánovas y Sánchez López, 2003). Por ejemplo, se podrían usar para predecir el peso del resultado de un test de velocidad de procesamiento en el rendimiento en una traducción contrarreloj.

Cuando el objetivo es explicar un fenómeno, se usan procedimientos para intentar establecer ciertas inferencias causales, siempre que se fundamenten en modelos teóricos muy estudiados, con «asociaciones suficientes entre dos variables, antecedentes temporales de la causa frente al efecto, falta de alternativas a las variables causales y una base teórica para la relación» (Amor Andrés y Sánchez-Elvira Paniagua, 2003: I80). Los diseños correlacionales causales de ecuaciones estructurales son los más frecuentes y permiten trabajar en el ajuste de los datos desde un modelo estadístico a un modelo teórico preestablecido.

Los análisis comparativos no permiten manipular variables (por ejemplo, no se pueden modificar los niveles de autoeficacia general de una persona) y obviamente no se pueden formar grupos mediante aleatorización. Valoran si exis- 
ten diferencias estadísticamente significativas al comparar puntuaciones medias que obtienen diferentes grupos en la variable dependiente de interés, mediante el estadístico T de Student o los análisis de variación ANOVA y MANOVA. Un ejemplo de este diseño sería formar tres grupos según el nivel de autoeficacia de los sujetos y comparar su grado de satisfacción con su rendimiento ante una traducción compleja.

En los diseños cuasi-experimentales sí se manipulan las variables, es decir, las condiciones de exposición, pero tampoco permiten aleatorización de sujetos. Se analiza la interacción entre fuentes de variación individual y la situación. Por ejemplo, se podría dividir a los sujetos en grupos según su grado de asertividad, con prueba de traducción para medir su rendimiento en dos condiciones, con acceso a documentación y sin documentación. Los métodos estadísticos utilizados son los mismos que los ya expuestos para los diseños comparativos.

\section{CONCLUSIONES}

A pesar de que los estudios empíricos sobre los procesos cognitivos del traductor han ido en aumento en los últimos años, tanto la metodología de investigación aplicada como los enfoques adoptados, inspirados en gran medida en otras disciplinas, y sobre todo en la psicología, se encuentran aún en proceso de consolidación. En el presente trabajo hemos presentado una aproximación de orientación psicológica al estudio de las diferencias individuales del traductor, que creemos puede contribuir a enriquecer ambos aspectos.

Como hemos visto, se trata de un campo que ya ha despertado el interés de los investigadores sobre traducción y que cuenta con antecedentes en nuestra disciplina, si bien muchos adolecen de ciertas carencias relacionadas con la atomi- zación, su escasa comparatividad, el poco uso del potencial estadístico y de instrumentos psicométricos adecuados, entre otros, en parte debidas a la ausencia de un marco metodológico sólido de investigación. Por ello, esperamos que el marco preliminar específico propuesto contribuya a impulsar esta rama de estudio y, sobre todo, a diversificar los modelos de investigación (por ejemplo, mediante la combinación de diferentes tipos de variables, diseños y ámbitos de variabilidad), y ampliar sus objetivos. Hasta ahora casi la totalidad de los estudios precedentes han sido de carácter descriptivo; el gran reto reside en la puesta en marcha de otros que tengan como objeto también predecir y explicar mediante un mejor aprovechamiento del potencial estadístico del que disponemos en la actualidad.

Entre otras vías de investigación, las conclusiones del presente trabajo sugieren que sería interesante avanzar en el afinamiento del marco metodológico de investigación de las diferencias individuales en traductología, idealmente a partir de la elaboración de metaanálisis cualitativos, pero también cuantitativos, de los trabajos publicados, algo esto último que hasta ahora no había sido posible precisamente por la ausencia de un marco de investigación común. En esta línea de trabajo, se podría caminar hacia el consenso sobre qué variables de análisis resultan prioritarias, qué diseños más adecuados dada su naturaleza o hacia un mayor control de las variables extrañas. Otro campo que requerirá de mayores esfuerzos en el futuro es el desarrollo de instrumentos psicométricos adaptados a las necesidades específicas de nuestra disciplina. Para terminar, cabría profundizar en las diferentes áreas de aplicación del estudio de las diferencias individuales en traductología, sobre todo en el contexto didáctico, para conseguir una mayor adaptación de los proyectos docentes 
al perfil del alumnado; e, incluso, en el ámbito laboral, logrando una mejor adecuación de los candidatos a los perfiles demandados mediante procesos de selección de personal.

RECIBIDO EN MAYO DE 2013

ACEPTADO EN SEPTIEMBRE DE 2013

VERSIÓN FINAL DE OCTUBRE DE 2OI3

\section{REFERENCIAS BIBLIOGRÁFICAS}

Alves, F. (ed.). (2003). Triangulating Translation: Perspectives in process oriented research, Amsterdam: John Benjamins.

Amirzehni, S. (2013). «The Relationship between the Translators's Personality and the Quality of Translation in Narrative Texts». International Conference on Interdisciplinary Translation Studies, 30 abril - 2 de mayo, Mashhad, Imam Reza Int'l University.

Amor Andrés, P.J.; Sánchez-Elvira Paniagua, A. (2003). «Métodos de investigación en el estudio de las diferencias individuales». En Sánchez-Elvira Paniagua, A. (ed.). Introducción al estudio de las diferencias individuales. Madrid: Sanz y Torres, pp. I46-192.

Andrés Pueyo, A. (1997). Manual de psicología diferencial, Madrid: McGraw Hill.

Angelone, E. (2010). «Uncertainty, Uncertainty Management and Metacognitive Problem Solving in the Translation Task». En Shreve, G. y Angelone, E. (eds.). Translation and Cognition. Ámsterdam: John Benjamins, pp. I7-40.

Angelone, E.; Shreve, G. (20II). «Uncertainty Management, Metacognitive Bundling in Problem Solving and Translation Quality». En O'Brien, Sh. (ed.). Cognitive Explorations of Translation. Londres: Continuum Studies in Translation, pp.Io8-130.

Asadi, P; Séguinot, C. (2005). «Shortcuts, Strategies and General Patterns in a Process Study of Nine Professionals». Meta: Translators'Journal, 5o/2, pp. 522-547.

Bajo, T.; Padilla, P.; Muñoz, R.; Padilla, F.; Gómez, C.; Puerta, M. C.; Gonzalvo, P.; Macizo, P. (20oI). «Comprehension and Memory Processes in Translation and Interpreting». Quaderns, Revista de Traducció, 6, pp. 27-3I.
Barboni, T. (I999). «Inconscient et traduction». En Lejeune, C. (ed.): Théorie et Pratique de la Traduction III, Mons: Le Ciephum, pp. 23-33.

Beeby, A.; Fernández, M.; Fox, O.; Hurtado Albir, A.; Kuznik, A.; Neunzig, W.; Rodríguez, P.; Romero, L.; Wimmer, S. (2OII). «Results of the Validation of the PACTE Translation Competence Model: Translation Project and Dynamic Translation Index». En Sharon O'Brien (ed.). Cognitive Explorations of Translation. Londres \& Nueva York: Continuum Studies in Translation, 30-53.

Binet, A.; Simon, Th. (1954). La mesure du développement de l'intelligence chez les jeunes enfants, Publications de la Sociéte Alfred Binet, París: Bourrelier.

Bolaños Medina, Alicia (2008). «Cohesión y sentimiento de eficacia colectiva en los grupos virtuales de aprendizaje colaborativo. Implicaciones didácticas». En I Jornadas Internacionales de Teleformación. Las Palmas de Gran Canaria: Universidad de Las Palmas de Gran Canaria.

- (2012). «Las pruebas psicométricas en la investigación empírica sobre los procesos cognitivos del traductor». En Cruces Collado, S.; Luna Alonso, A.; Del Pozo Triviño, M. y Álvarez Lugrís, A. (eds.). Traducir en la Frontera. Actas del IV Congreso de AIETI. Granada: Atrio, pp. 469-482.

- (2013). «La tolerancia a la ambigüedad y los procesos cognitivos del traductor» [en prensa].

Bontempo, K. ; Loggerenberg, V. (2OII). «Interpreters and Coping Strategies for Managing Occupational Stress». Synergise! Conference Lectures Series [DVD]. Sydney: Australian Institute of Interpreters and Translators.

Bontempo, K. ; Napier, J. (20II). «Evaluating Emotional Stability as a Predictor of Interpreter Competence and Aptitude in Interpreting». En Shlesinger, M. y Pöchhacker, F. (eds.). Aptitude for Interpreting: Special issue on Interpreting, I2/I, pp. 85-105.

Booth-Kewley, S.; Friedman, H. S. (1987). «Psychological Predictors of Heart Disease: A Quantitative Review». Psychological Bulletin, Ior/3, pp. 343-362.

Bowers, K. (1973). «Situationism in Psychology: An Analysis and Critique». Psychological Review, 80, pp. 307-336.

Carl, M.; Dragsted, B.; Lykke-Jakobsen, A. (20II). «A Taxonomy of Human Translation Styles». 
Translation Journal, 16/2, <http://translationjournal.net/journal/56taxonomy.htm> [Consulta: 15 de febrero de 20I3].

Cattell, R. B. (1946). The Description and Measurement of Personality, Nueva York: World Book.

Colom Marañón, R. (1998). Psicología de las diferencias individuales. Teoría y práctica, Madrid: Pirámide.

Darwin, C. [1872] (1998). The Expression of Emotions in Man and Animals, Nueva York: Oxford.

Eysenck, H.; Eysenck, M. (1985). Personalidad y diferencias individuales, Madrid: Pirámide.

Fernández Sánchez, M.; Muñoz-Martín, R. (2008). Aproximaciones cognitivas al estudio de la traducción y la interpretación, Granada: Comares.

Fontes de Gracia, S.; García-Gallego, C.; Quintanilla Cobían, L.; Rodríguez Fernández, R.; Rubio de Lemus, P.; Sarriá Sánchez, E. (eds.). (2010). Fundamentos de investigación en psicologia, Madrid: UNED.

Forer, B. R. (1949). «The Fallacy of Personal Validation: A Classroom Demonstration of Gullibility». Journal of Abnormal and Social Psychology, 44, pp. II8-I23.

Froeliger, N. (2004). «Les mécanismes de la confiance en traduction - aspects relationnels». Journal of Specialised Translation, 2, pp. 52-6o.

Galton, F. (1883). Inquiries into the Human Faculty and its Development, Londres: J. M. Dent.

Hansen, G. (2003). "Controlling the Process. Theoretical and Methodological Reflections on Research in Translation Processes». En Alves, F. (ed.). Triangulating Translation: Perspectives in Process Oriented Research. Amsterdam: John Benjamins, pp. $25^{-42}$.

Henderson, J. (1987). Personality and the Linguist: A Comparison of the Personality Profiles of Professional Translators and Interpreters, Bradford: University of Bradford Press.

Hjort-Pedersen, M.; Faber, D. (2009). «Uncertainty in the Cognitive Processing of a Legal Scenario: A Process Study of Student Translators». Hermes, 42, pp. 189-2Io.

House, J. (2000). "Consciousness and the Strategic Use of Aids in Translation». En TirkkonenCondit , S. y Jääskeläinen, R. (eds.). Tapping and Mapping the Processes of Translation and Interpreting. Outlooks on Empirical Research. Amsterdam: John Benjamins, pp. I49-I62.
Huarte de San Juan, J. [1575] (1976). Examen de genios para las ciencias, Madrid: Espasa Calpe.

Hubscher-Davidson, S. (2007). «An Empirical Investigation into the Effects of Personality on the Performance of French to English Student Translators». Tesis doctoral. University of Bath.

- (2009). «Personal Diversity and Diverse Personalities in Translation: a Study of Individual Differences». Perspectives: Studies in Translatology, $\mathrm{I} 7 / 3$, pp. I75-I92.

- (2013). «Emotional Intelligence and Translation Studies: A New Bridge». Meta: Translator's Journal, 58/2, pp. 324-346.

Hurtado Albir, A. (200I). Traducción y traductología: introducción a la traductología, Madrid: Cátedra.

Jääskeläinen, Riita (2000). «Focus on Methodology in Think-Aloud Studies on Translating». En Tirkkonen-Condit, S. y Jääskeläinen, R. (eds.). Tapping and Mapping the Processes of Translation and Interpreting. Outlooks on Empirical Research. Amsterdam: John Benjamins, pp.7I-82.

Jääskeläinen, Riitta. 20I2. "Translation Psychology». En Gambier, Y. y van Doorslaer, L. (eds.). Handbook of Translation Studies (vol. 3). Amsterdam/ Philadelphia: John Benjamins, pp. I9I-I97.

Jung, C. G. (I92I). Psychologische Typen, Zurich: Rascher Verlag.

Kiraly, D. (I997). «Think-Aloud Protocols and the Construction of a Professional Translator SelfConcept». En Danks, J. H.; Shreve, G.; Fountain, S. B. y McBeath, M. K. (eds.). Cognitive Processes in Translation and Interpreting. Thousand Oaks: Sage, pp. 137-160.

Kline, P. (I996). «Personality Tests». En Hampson, S. y Colman, M. (eds.). Individual Differences and Personality. Londres/Nueva York: Longman, pp. 77-98.

Künzli, A. (2004). «Risk Taking: Trainee Translators vs Professional Translators. A Case Study». Journal of Specialised Translation, 2, pp. 34-49.

Kussmaul, P. (I995). Training the translator, Amsterdam/Philadelphia: John Benjamins.

Kussmaul, P.; Tirkkonen-Condit, S. (1995). «ThinkAloud Protocol Analysis in Translation Studies». TTR: traduction, terminologie, rédaction, 8/I, pp. I77-199.

Laukkanen, J. (1996). "Affective and Attitudinal Factors in Translation Processes». Target, 8/2, pp. 257-274. 
Magnusson, D.; Endler, N. S. (eds.) (I977). Personality at the Crossroads, Hilsdale: L. Erlbaum.

Marín García, Á. (2005). «Creatividad y automatización al traducir». Proyecto de investigación dirigido por Muñoz Martín, R., Universidad de Granada.

McCrae, R.; Costa, P. T. (I989). «Reinterpreting the Myers-Briggs Type Indicator From the Perspective of the Five-Factor Model of Personality». Journal of Personality, 57/I, pp. I7-40.

Mischel, W.; Shoda, Y. (I995). «A cognitive Affective System Theory of Personality: Reconceptualizing Situations, Dispositions, Dynamics, and Invariance in Personality Structure». Psychological Review, I02, pp. 246-248.

Muñoz-Martín, R. (2009). "The Way they Were: Subject Profiling in Translation Process Research». En Mees, I.; Alves, F y Göpferich, S. (eds.). Methodology, Technology and Innovation in Translation Process Research: A Tribute to Arnt Lykke Jakobsen. Copenhague: Samfundslitteratur Press, pp. 87-108.

O'Brien, Sharon (ed.) (20II). Cognitive Explorations of Translation, Londres: Continuum International Publishing Group.

Olmedo Montes, M.; Sánchez-Elvira Paniagua, A. (2003). «Desarrollo histórico del estudio de las diferencias individuales I: etapa precientífica y establecimiento de la psicología diferencial como disciplina científica». En Sánchez-Elvira Paniagua, A. (ed.). Introducción al estudio de las diferencias individuales. Madrid: Sanz y Torres, pp. 3-51.

Prassl, F. (20I0). «Translators' Decision-making Processes in Research and Knowledge Integration». En Göpferich, S.; Alves, F.; Mees and I. M. (eds.). New Approaches in Translation Process Research. Copenhagen Studies in Language, 39. Copenhague: Samfundslitteratur, pp. 57-82.

Presas Corbella, M. (2006). «El estudio de la competencia traductora: de la observación empírica a las tareas experimentales». XII Congreso Latinoamericano de Estudios Germanísticos, La Habana, <http://grupsderecerca.uab.cat/pacte/ sites/grupsderecerca.uab.cat.pacte/files/2006_ PACTE_CUBA_PRESENTACION.pdf> [Consulta: 25 de marzo de 20I3].
Pym, A. (2005). «Explaining Explicitation». En Károly, K. (ed.). New Trends in Translation Studies. In Honour of Kinga Klaudy. Budapest: Akadémiai Kiadó, pp. 29-43.

Reiss, K.[I97I] (2000). Translation Criticism. Manchester: St. Jerome Publishing.

Rodrigues, C. (2002). «A abordagem processual no estudo da tradução: uma meta-análise qualitativa». Cadernos de Tradução 2/Io, pp. 23-57.

Rojo López, A. (2013). Diseños y métodos de investigación en traducción. Madrid: Síntesis.

Royce, J. R.; Powell, A. (1983). Theory of Personality and Individual Differences. Factors, Systems and Processes, Englewood Cliffs: Prentice Hall.

Sánchez Cánovas, J.; Sánchez López, M. P. (I999). Psicologia de la diversidad humana, Madrid: Centro de Estudios Ramón Areces.

Sánchez-Elvira Paniagua, A.; Olmedo Montes, M. (2003). «Marco conceptual de la psicología de las diferencias individuales». En Sánchez-Elvira Paniagua, A. (ed.). Introducción al estudio de las diferencias individuales. Madrid: Sanz y Torres, pp. IO3-I44.

Skehan, P. I99I. "Individual Differences in Second Language Learning». Studies in Second Language Acquisition, I3/2, pp. 275-298.

Spearman, C. (I9I4). «The Theory of Two factors». Psychological Review, 2I, pp. IOI-II5.

Spranger, E. (1920). Lebensformen. Geisteswissenschaftliche Psychologie und Ethik der Persönlichkeit, Halle: M. Niemeyer.

Tirkkonen-Condit, S. (1996). «What Is in the Black Box? Professionality in Translational Decisions in the Light of TAP Research». En Lauer, A.; Gerzymisch-Arbogast, H.; Haller, J. y Steiner, E. (eds.). Übersetzungswissenschaft im Umbruch. Festschrift für Wolfram Wilss zum 70 Geburtstag. Tübigen: Narr, 251-257.

Tirkkonen-Condit, S. 2000. «Uncertainty in Translation processes». En Tirkkonen-Condit, S. y Jääskeläinen, R. (eds.). Tapping and Mapping the Processes of Translation and Interpreting. Outlooks on Empirical Research. Amsterdam: John Benjamins, pp. I23-I42.

Wilss, W. (1996). Knowledge and Skills in Translator Behavior. Amsterdam y Philadelphia: John Benjamins. 